\title{
A NEW STATISTICAL IMAGE RECONSTRUCTION ALGORITHM FOR POLYENERGETIC X-RAY CT
}

\author{
Mónica Abella ${ }^{1}$, Jeffrey A. Fessler ${ }^{2}$ \\ ${ }^{1}$ Unidad de Medicina y Cirugía Experimental, Hospital General Universitario Gregorio Marañón, Spain \\ ${ }^{2}$ EECS Dept., The University of Michigan, USA
}

\begin{abstract}
This paper presents a new statistical reconstruction algorithm for X-ray CT. The algorithm is based on Poisson statistics and a physical model that accounts for the measurement nonlinearities caused by energy-dependent attenuation. We model each voxel's attenuation as a mixture of bone and soft tissue by defining density-dependent tissue fractions, maintaining one unknown per voxel avoiding the need of a pre-segmentation. Rather than requiring the entire $\mathrm{X}$-ray spectrum, the method approximates the $2 \mathrm{D}$ beam hardening function corresponding to bone and soft tissue with the 1D function corresponding to water and one or two empirical tuning parameters.

Results on simulated human data (NCAT phantom) showed a beam hardening reduction similar to conventional post-processing techniques, but with an improved signal to noise ratio.
\end{abstract}

Index Terms - X-ray computed tomography, beam hardening, penalized-likelihood image reconstruction

\section{INTRODUCTION}

Filtered back projection (FBP) is the reconstruction method traditionally used in X-ray CT. However, non-radon scanning geometries, such as cone-beam and multi-slice helical CT, the desire for lower-dose scans, and the necessity of handling truncated data, are increasingly challenging the capabilities of FBP. Statistical techniques have better bias-variance performance, permit lower dose and accommodate models of data acquisition physics such as beam hardening, scatter, partial-volume effects, etc [1].

Beam hardening is a process whereby the average energy of the X-ray beam increases as the beam propagates through a material because lower energy $\mathrm{X}$-rays are preferentially attenuated. X-rays traversing different paths through an object will emerge with different spectra, leading to data inconsistencies (in the Radon sense) and reconstruction artifacts: 'cupping' due to water-related beam-hardening and 'streaks' or 'shadows', and 'spill over' of bone areas into soft tissue, due to bone-related beam-hardening [2-4]. Several schemes have been proposed to correct for such artifacts. Most scanners use the "water correction method" that assumes that all the materials in the scan field are water equivalent in X-ray attenuation characteristics and corrects the measurements prior to reconstruction $[2,5,6]$. This is often not sufficient for non homogeneous objects, especially in the presence of high density areas, like bone, leading to suboptimal correction.

Dual-energy imaging (typically $80 \mathrm{kVp}$ and $120 \mathrm{kVp}$ in human scans) [7-9] doubles the number of measurements and enables reconstruction of separate bone and soft tissue images. It is useful for tissue characterization and quantitative CT, but its major drawback is the need for two separate scans, requiring a sophisticated hardware setup. It can also significantly increase the radiation dose.

Joseph and Spital [4] described a post processing technique that involves segmenting the bone voxels from an initial FBP reconstruction, which are then forward projected. This bone projection provides an estimate of the amount of nonlinear beam hardening distortion, which is then corrected for [4, 10-12].

Several iterative methods have been proposed for beam hardening correction. Yan et al [13] developed an iterative, but non-statistical, beam hardening correction method, assuming two categories of materials and iteratively computing their volume fraction at each pixel. DeMan et al [1] proposed a statistical approach modeling the object attenuation as a linear combination of the attenuation properties of two base substances. The algorithm needs knowledge of the polyenergetic source spectrum but does not need a pre-segmented image. Elbakri and Fessler [14] presented a statistical algorithm that, like the Joseph and Spital method, required pre-segmenting an initial image and did not permit pixels to contain tissue mixtures. Later [15], they eliminated the necessity of segmentation and allowed pixels to contain mixtures. Both approaches needed tabulated measurements of the line integrals of bone and water over a reasonable range of object thicknesses for the CT system of interest. To avoid modeling the entire X-ray spectrum, Srivastava and Fessler [16] proposed a simplified statistical image reconstruction approach for polyenergetic X-ray CT using the using the same calibration data and tuning parameters as Joseph and Spital. However, that 
method uses approximations that can lead to nonphysical negative values that can cause convergence problems.

In this paper, we explore in more depth the latter work, proposing improved approximation functions and presenting a segmentation free algorithm by modeling the tissue fractions in the voxels as functions of the density [15].

The proposed statistical reconstruction algorithm includes beam hardening correction without requiring the full X-ray spectrum and eliminates the segmentation step needed in most other approaches. The algorithm iteratively minimizes the Poisson likelihood. Although one version of the algorithm can be guaranteed to ensure monotonicity, we usually use ordered subsets to accelerate the algorithm, compromising it. Including scatter estimates in the algorithm is straightforward.

We tested the algorithm on simulated human data using the CT part of the NURBS-based Cardiac-Torso (NCAT) phantom [17].

\section{MATERIALS AND METHODS}

We model the measurements as independently distributed Poisson random variables [18] that are contaminated by extra background counts caused primarily by scatter:

$$
Y_{i} \sim \text { Poisson }\left\{\bar{Y}_{i}\right\}, \quad i=1, \ldots, N,
$$

where

$$
\bar{Y}_{i}=\int I_{i}(\varepsilon) e^{-\int_{L i} \mu(x, y, \varepsilon)} d \varepsilon+r_{i}
$$

where $\mu(x, y, \varepsilon)$ is the unknown energy-dependent attenuation map of the object. The integral in the exponent is taken over the line $L_{i}$ and $I_{i}(\varepsilon)$ incorporates the energy dependence of both the incident ray and the detector sensitivity. The term $r_{i}$ can account for scatter and other background signals.

\subsection{Segmentation free implementation}

We express the attenuation coefficient using the mass attenuation coefficient/density model:

$$
\mu_{j}(\varepsilon)=\sum_{k=1}^{K} m_{k}(\varepsilon) f_{k}^{j} \rho_{j},
$$

where the tissue fraction, $f_{k}^{j}$, is a unitless fraction that describes the contribution of material $k$ to attenuation in pixel $j$. Using this model directly would require $K$ sets of unknowns. Often one assumes $K=2$, i.e., the object is composed of only two materials: bone and soft tissue. Dualenergy scanning accommodates $K=2$ by providing a proportional increase in the available measurements. In single-energy scanning a common approach to reduce the number of unknowns is to assume that each pixel is either bone or soft tissue, i.e., $f_{k}^{j}$ is a binary function that is equal to 1 for pixels of tissue type $k$ and 0 for the rest. Usually the values $f_{k}^{j}$ are determined by segmenting a preliminary reconstruction $[14,16]$. Here we consider an alternative approach [15] that avoids segmentation by modeling the tissue fraction value in the pixel as a function of the estimated density in that pixel. For the two-material case we assume

$$
\begin{aligned}
& \mu_{j}(\varepsilon) \approx \sum_{k=1}^{2} m_{k}(\varepsilon) \rho_{j} f_{k}^{j}\left(\rho_{j}\right) \\
& =\left(m_{\mathrm{s}}(\varepsilon) f_{\mathrm{s}}^{j}\left(\rho_{j}\right)+m_{\mathrm{b}}(\varepsilon) f_{\mathrm{b}}^{j}\left(\rho_{j}\right)\right) \rho_{j} .
\end{aligned}
$$

The tissue fraction functions, $f_{\mathrm{s}}^{j}\left(\rho_{j}\right)$ and $f_{\mathrm{b}}^{j}\left(\rho_{j}\right)$ are built as explained in [15] and $f_{\mathrm{s}}^{j}\left(\rho_{j}\right)+f_{\mathrm{b}}^{j}\left(\rho_{j}\right)=1$. The contribution of each tissue type to the line integral along the $j$ th ray is now

$$
\begin{gathered}
t_{\mathrm{s}}(\rho)=\sum_{j=1}^{p} a_{i j} f_{\mathrm{s}}^{j}\left(\rho_{j}\right) \rho_{j}, \\
t_{\mathrm{b}}(\rho)=\sum_{j=1}^{p} a_{i j} f_{\mathrm{b}}^{j}\left(\rho_{j}\right) \rho_{j}=\sum_{j=1}^{p} a_{i j}\left(1-f_{\mathrm{s}}^{j}\left(\rho_{j}\right)\right) \rho_{j},
\end{gathered}
$$

where $a_{i j}$ denotes elements of the system matrix.

\subsection{Forward model}

The mean of the measured data along the path $L_{i}$ is then

$$
\begin{array}{r}
\bar{Y}_{i}(\rho)=\int I_{i}(\varepsilon) e^{-m_{\mathrm{s}}(\varepsilon) t_{\mathrm{s}}(\rho)-m_{\mathrm{b}}(\varepsilon) t_{\mathrm{b}}(\rho)} d \varepsilon+r_{i}= \\
I_{i} e^{-F\left(t_{s}^{i}(\rho), t_{b}^{i}(\rho)\right)}+r_{i},
\end{array}
$$

where

$$
I_{i} \equiv \int I_{i}(\varepsilon) d \varepsilon
$$

and the function $F$, that characterizes the beam hardening, is defined by

$$
F\left(t_{\mathrm{s}}, t_{\mathrm{b}}\right)=-\log \left(\int \frac{I(\varepsilon)}{I} \exp \left(-m_{\mathrm{s}}(\varepsilon) t_{\mathrm{s}}-m_{\mathrm{b}}(\varepsilon) t_{\mathrm{b}}\right) d \varepsilon\right),
$$

where we drop the dependence on ray $i$ for simplicity.

\subsection{Beam hardening function approximations}

The beam-hardening function $F\left(t_{s}, t_{b}\right)$ could be calculated if the X-ray spectrum were known, but often it is not. Tabulating $F$ experimentally is cumbersome. The function that is more easily measured and tabulated is the one that characterizes the beam hardening for only one tissue type, defined by

$$
F_{k}(t)=-\log \int \frac{I(\varepsilon)}{I} \exp \left(-m_{k}(\varepsilon) t_{k}\right) d \varepsilon .
$$

The table corresponding to water (equivalent to soft tissue) is available in most scanners. This table is used for the so-called water correction, though it is often insufficient for inhomogeneous objects. The method developed by Joseph and Spital [4] improves upon the water correction 
method and uses only one or two additional parameters that can be tuned empirically rather than requiring the entire $\mathrm{X}$ ray spectrum. The idea is based on the concept of "effective density," that is, that equivalent to water. Using this concept, we rewrite the beam hardening function as

$$
F\left(t_{\mathrm{s}}, t_{\mathrm{b}}\right)=F\left(t_{\mathrm{s}}+\sigma\left(t_{\mathrm{s}}, t_{\mathrm{b}}\right), 0\right)=F_{\mathrm{s}}\left(t_{\mathrm{e}}\right),
$$

where the water function is $F_{\mathrm{s}}\left(t_{\mathrm{s}}\right)=F\left(t_{\mathrm{s}}, 0\right)$ and we define

$$
\sigma\left(t_{\mathrm{s}}, t_{\mathrm{b}}\right) \equiv F_{\mathrm{s}}^{-1}\left(F\left(t_{\mathrm{s}}, t_{\mathrm{b}}\right)\right)-t_{\mathrm{s}}
$$

The line integral of the effective density (water equivalent) is given by

$$
t_{\mathrm{e}}=t_{\mathrm{s}}+\sigma\left(t_{\mathrm{s}}, t_{\mathrm{b}}\right)
$$

An exact expression for $\sigma\left(t_{s}, t_{b}\right)$ would require the full $\mathrm{X}$ ray spectrum, just like the function $F\left(t_{s}, t_{b}\right)$. What would be preferable is an approximation involving only one or two tuning parameters that one can determine experimentally. Fig. 1 shows $\sigma\left(t_{s}, t_{b}\right)$ for a typical polyenergetic spectrum.
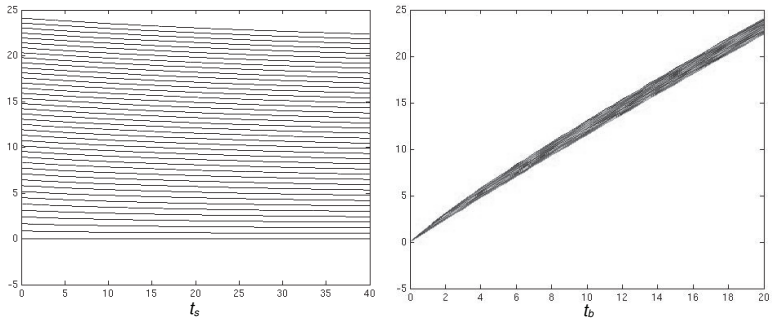

Fig. 1. Example of $\sigma\left(t_{s}, t_{b}\right)$. Left: profile versus $t_{s}$ for different values of $t_{b}$. Right: profile versus $t_{b}$ for different values of $t_{s}$.

Fig. 1 shows that for small bone areas, the dependence on $t_{s}$ is negligible when the amount of bone in the object is small, thus, we can approximate $\sigma\left(t_{s}, t_{b}\right)$ by a function that depends only on $t_{b}$. One possible approximation, suggested in [4], is based on the power series approximation of the function that defines the measured projection. This is a similar approach to the one followed by Hsieh [11], which results in

$$
\hat{\sigma}_{1}\left(t_{\mathrm{s}}, t_{\mathrm{b}}\right)=A t_{\mathrm{b}}-B t_{b}^{2} .
$$

Although this approximation works adequately in an analytic reconstruction scheme, it fails when included in the forward model of an iterative algorithm because it can yield negative values. Furthermore, one can show mathematically that $\sigma\left(t_{s}, t_{b}\right)$ is a monotone increasing function of both of its arguments, whereas (14) is not. To overcome this problem, we investigated the following alternative approximation:

$$
\hat{\sigma}_{2}\left(t_{\mathrm{s}}, t_{\mathrm{b}}\right)=\frac{A t_{\mathrm{b}}}{1+\frac{B}{A} t_{\mathrm{b}}} .
$$

We also explored another approximation that better matches the true shape of $\sigma\left(t_{s}, t_{b}\right)$, but requires one more parameter:

$$
\hat{\sigma}_{3}\left(t_{\mathrm{s}}, t_{\mathrm{b}}\right)=-\log \left(\alpha \exp \left(-\beta_{1} t_{\mathrm{b}}\right)+(1-\alpha) \exp \left(-\beta_{2} t_{\mathrm{b}}\right)\right)
$$

Substituting (15) or (16) into (11) and (7) yields our proposed forward model.

\subsection{Algorithm}

The negative log-likelihood for independent Poisson measurements is given by

$$
-L(\rho)=\sum_{i=1}^{N} h_{i}\left(F_{s}\left(t_{e}(\rho)\right)\right)
$$

where,

$$
h_{i}(l)=-Y_{i} \log \left(I_{i} e^{-l}+r_{i}\right)+I_{i} e^{-l}+r_{i} .
$$

Given that data is noisy and it is an ill-posed problem, we include regularization by adding a penalty term to the likelihood function that controls how much the object $\rho$ departs from our assumptions about image properties. In this work we used a 3D roughness penalty function with the convex edge-preserving Huber potential. To improve resolution uniformity, we modified the penalty function as described in [19]. With regularization we have the following penalized cost function:

$$
\Phi(\rho)=-L(\rho)+\beta R(\rho),
$$

where $\beta$ is a scalar that controls the tradeoff between the data-fit and the penalty terms. A small value will result in very good spatial resolution in the absence of noise, but very noisy estimates in the presence of noise. Conversely, a large value will emphasize minimizing $R(x)$, which usually means a smooth estimate with low noise.

We can derive an iterative algorithm using the principles of optimization transfer. This results in the following update:

$$
\rho^{n+1}=\rho^{n}-D^{-1} \nabla \Phi\left(\rho^{n}\right)
$$

where $D$ is a diagonal matrix that influences the rate of convergence. We can design $D$ to ensure the algorithm monotonically decreases the cost function. However, following [14] usually we choose the elements of $D$ approximately by using the pre-computed curvature:

$$
d_{j}=m_{\mathrm{s}}^{2}\left(\varepsilon_{e f f}\right) \sum_{i=1}^{N} a_{i j}\left(\sum_{j} a_{i j}\right) Y_{i} .
$$

\section{RESULTS}

Fig. 2 shows the results on one slice of the NCAT phantom. The proposed method eliminates the beam hardening artifact while showing a significant reduction of noise when compared with the post-processing method by Joseph and Spital (JS). The reduction of noise was of $40.5 \%$ in the round ROI on the tissue area shown in Fig. 2-A, while substantially reducing noise in the background. 


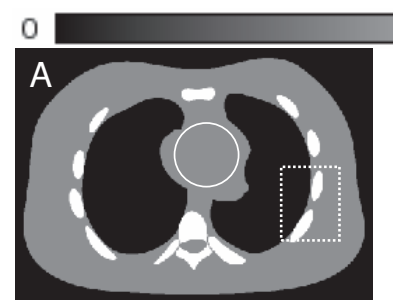

Original image

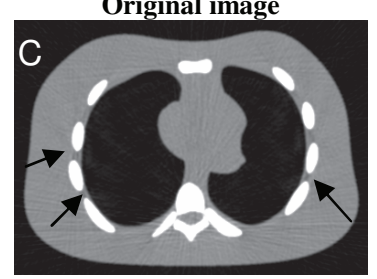

FDK + Water correction

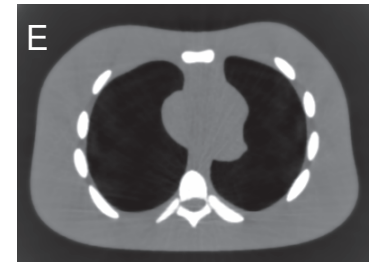

Statistical algorithm (no BH)
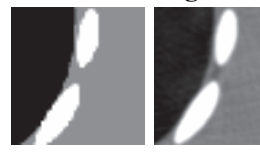

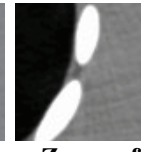

Zoom of images $A-$
2.1

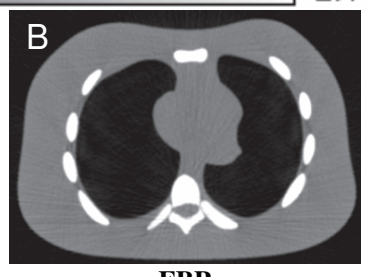

FBP

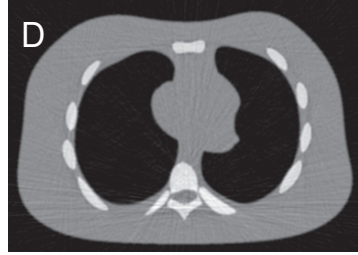

FBP + JS correction

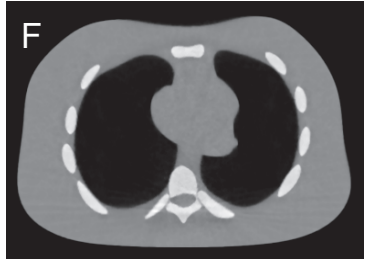

Statistical algorithm proposed

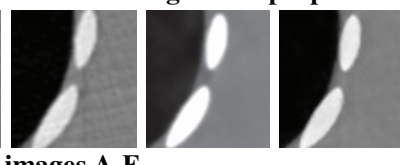

Fig. 2. Reconstructed $512 \times 512$ pixel transaxial slice of the NCAT phantom. The bottom images are a zoom of the area inside the dotted square drawn in A.

\section{SUMMARY}

We have presented present a statistical reconstruction algorithm based on minimizing the negative Poisson loglikelihood with regularization. The method includes beamhardening correction but requires only the water-correction function and one or two tuning parameters instead of requiring the entire $\mathrm{X}$-ray spectrum. The method also eliminates the segmentation step needed in most other approaches. Although one version of the algorithm is monotonic, we usually use ordered subsets to accelerate the algorithm, forgoing monotonicity. Including scatter estimates in the algorithm is straightforward.

The algorithm outperforms the commonly used water correction method, achieving a similar beam hardening correction as the post-processing technique proposed by Joseph and Spital, but with increase signal-to-noise ratio and eliminating the need of pre-segmentation.

The approximations to $\sigma\left(t_{s}, t_{b}\right)$ used here are accurate when there are only small areas of bone. In future work we will explore more accurate approximations by considering the dependence on $t_{s}$. We will also apply the algorithm to real data.

\section{REFERENCES}

[1] B. De Man, J. Nuyts, P. Dupont, G. Marchal, and P. Suetens, "An Iterative Maximum-Likelihood Polychromatic Algorithm for CT", IEEE Trans Med Imag, vol. 20, no. 10, pp. 999-1008, 2001.

[2] R.A. Brooks and G.D. Chiro, "Beam hardening in x-ray reconstruction tomography", Phys Med Biol, vol. 21, pp. 390-8, 1976.

[3] A.C. Kak and M. Slaney, Principles of Computerized Tomographic Imaging, IEEE Press, New York, 1988.

[4] P.M. Joseph and R.D. Spital, "A method for correcting bone induced artifacts in computed tomography scanners", J Comp Assisted Tomo, vol. 2, no. 1, pp. 100-8, 1978.

[5] G.T. Herman, "Correction for beam hardening in computed tomography", Phys Med Biol, vol. 24, no. 1, pp. 81-106, 1979.

[6] W.D. McDavid, R.G. Waggener, W.H. Payne, and M.J. Denis, "Correction for spectral artifacts in cross-sectional reconstruction from X-rays", Med Phys, vol. 4, no. 1, pp. 54-7, 1997.

[7] R.E. Alvarez and A. Macovski, "Energy-selective reconstruction in x-ray computerized tomography", Phys Med Biol, vol. 21, no. 5, pp. 733-44, 1976.

[8] J.A. Fessler, I.A. Elbakri, P. Sukovic, and N.H. Clinthorne, "Maximum-likelihood dual-energy tomographic image reconstruction", Proc SPIE, vol. 4684, no. 1, pp. 38-49, 2002.

[9] P. Sukovic and N.H. Clinthorne. "Design of an experimental system for dual energy x-ray CT", IEEE Nucl Sci Symp, vol. 2, pp. 1021-2, 1999.

[10] P.M. Joseph and C. Ruth, "A method for simultaneous correction of spectrum hardening artifacts in CT images containing both bone and iodine", Med Phys, vol. 24, no. 10, pp. 1629-34, 1997.

[11] J. Hsieh, R.C. Molthen, C. A. Dawson, and R.H. Johnson, "An iterative approach to the beam hardening correction in cone beam CT", Med Phys, vol. 27, no. 1, pp. 23-29, 2000.

[12] O. Nalcioglu and R.Y. Lou, "Post-reconstruction Method for Beam Hardening in Computerised Tomography", Phys Med Biol, vol. 24, no. 2, pp. 330-40, 1979.

[13] C.H. Yan, R.T. Whalen, G.S. Beaupré, S.Y. Yen, and S. Napel, "Reconstruction algorithm for polychromatic CT imaging: application to beam hardening correction", IEEE Trans Med Imag, vol. 19 , pp. 1-11, 2000.

[14] I.A. Elbakri and J.A. Fessler, "Statistical Image Reconstruction for Polyenergetic X-Ray Computed Tomography", IEEE Trans Med Imag., vol. 21, no. 2, pp. 89-99, 2002.

[15] I.A. Elbakri and J.A. Fessler, "Segmentation-free statistical image reconstruction for polyenergetic $\mathrm{x}$-ray computed tomography with experimental validation", Phys Med Biol, vol. 48, no. 15, pp. 2453-77, 2003.

[16] S. Srivastava and J.A. Fessler. "Simplied statistical image reconstruction algorithm for polyenergetic X-ray CT", IEEE Nucl Sci Symp, vol. 3, pp. 23-9, 2005.

[17] W.P. Segars, D.S. Lalush, and B.M.W. Tsui, "A realistic spline-based dynamic heart phantom", IEEE Trans Nucl Sci, vol. 46, no. 3, pp. 503-6, 1999.

[18] H. Erdogan and J.A. Fessler, "Monotonic algorithms for transmission tomography", IEEE Trans Med Imag, vol. 18, no. 9, pp. 801-14, 1999.

[19] J.A. Fessler and W.L. Rogers, "Spatial Resolution Properties of Penalized-Likelihood Image Reconstruction: Space-Invariant Tomographs", IEEE Trans Imag Proc, vol. 5, no. 9, pp. 1346-58, 1996. 